\title{
Modelling of a compact anisotropic star as an anisotropic fluid sphere in $f(T)$ gravity
}

\author{
D. Momeni ${ }^{a}$, G. $\operatorname{Abbas}^{b}$, S. Qaisar ${ }^{c}$, Zaid Zaz ${ }^{d}$, R. Myrzakulov ${ }^{e}$ \\ a Department of Physics, College of Science, \\ Sultan Qaboos University, P.O. Box 36, \\ P.C. 123, Al-Khodh, Muscat, Sultanate of Oman \\ $b$ Department of Mathematics, \\ The Islamia University of Bahwalpur, Pakistan \\ ${ }^{c}$ Department of Mathematics, \\ COMSATS Institute of Information Technology Sahiwal-57000, Pakistan \\ ${ }^{d}$ Department of Electronics and Communication Engineering, \\ University of Kashmir, Srinagar, Kashmir-190006, \\ India. e Eurasian International Centre for Theoretical Physics and \\ Department of General $\&$ Theoretical Physics, \\ Eurasian National University, \\ Astana 010008, Kazakhstan
}

\begin{abstract}
In this article, authors have discussed the new exact model of anisotropic star in $f(T)$ theory of gravity. The parametric form of metric functions has been implemented to solve the dynamical equations in $f(T)$ theory with the anisotropic fluid. The novelty of the work is that the obtained solutions do not contain singularity but potentially stable. The estimated values for mass and radius of the different strange stars RX J 1856-37, Her X-1, and Vela X-12 have been utilized to figure the values of unknown constants in Krori and Barua metric. The physical parameters like anisotropy, stability and redshift of the stars have been examined in detail.
\end{abstract}

PACS numbers: 04.50.Kd, 04.70.Dy.

\section{INTRODUCTION}

General Relativity (GR) is a classical gauge theory to describe long range interaction between gravitational objects. It alone can also account for the accelerated expansion of the universe using a fine- tuned cosmological constant term $\Lambda[1]-[5]$ or quintessence scalar field as dark energy [6],[7] or quintal fields [8]. However the existence of dark energy requires the introduction of additional fluids capable of dominating over the standard pressure less matter [9]. There is a simpler course to get around dark energy and to consistently explain the accelerated expansion. This is done by considering a cosmological constant. However the necessary expected value of the cosmological constant must be extremely larger than the observed value. This is due to quantum considerations [10]. There however is an internally consistent approach to account for the accelerated expansion of the universe. This is based on addressing the cosmic dynamics by exploring further degrees of freedom of the gravitational field. In other words to account for the accelerated dynamics of the universe, the Einstien Hilbert action of general relativity is modified [11]-[13]. Recently a different but interesting modification introduced as Gravitys Rainbow models [14] as an extension of the doubly special relativity theory [15], in which several interesting features have been studied like absence of effective horizon in black holes, black rings, mini black holes, gravitational collapse, brane solutions and others [16].

An extensively studied approach to modified gravity stems from the "Teleparallel equivalent to General Relativity" (TEGR) [17],[18]. It is noteworthy that unlike general relativity to explain the gravitational effects TEGR uses an anti symmetric connection equipped with a non-vanishing torsion and a zero curvature (Weitzenbock connection). In other words TEGR uses torsion to explain gravitational effects unlike General relativity which uses curvature. It may be moted that in order to define the Weitzenbock connection in TEGR tetrad fields are used as dynamical variables [19].

Using TEGR a straight forward modification of the Einstien Hilbert action may be performed by replacing the scalar torsion with an arbitrary smoooth function of the scalar torsion $f(T)$. This class of teleparallel theories is known as $f(T)$ gravity (see [20] and refrences in for a comprehensive review). Accelerated expansion of the cosmic Hubble parameter as well as dark matter can be explained using $f(T)$ gravity [21]-[48].

In gravitational theories, localized and positive valued mass densities could suddenly collapse, emerging thermodynamics that can produce a hydrodynamic pressure impose on the mass distribution. If the collapse of the matter distribution is continued then mass contents are transformed into a compact star. The compact star has a finite size $R \sim 10 \mathrm{Km}$ and a huge amount of mass of order of solar mass $M \sim 1.4 M_{\odot}$ [49]. A type of compact star is neutron star, it has very strong surface gravity and conequently a strongly coupled regime of thermodynamics. A way to detect these massive objects is by looking at the Doppler shift in spectral lines emitted by atoms in the surface of the candidated stars. One can find not much gravity on the earth, more on the compact star because surface gravity 
of such stars is of order $\kappa=2 \times 10^{11}$. Except than this big surface gravity, there is a greater difference between individual plants which makes the species and neutron stars. A reason is that the gravitational field from compact stars, have both electric and magnetic components and contains a definite amount of electromagnetic energy and thanks to some basic parameters, for example emission above-ground level,the electromagnetic effects are stronger than thermodynamic effects. It was demonstrated that the thermodynamic parameters, namely pressure $p$, energy density $\rho$, the mass function $M$ and radius $R$ are related according to a set of state equations, called as Tolman-OppenheimerVolkoff (TOV) equations in GR [50]-[52]. TOV equation is a set of first order, non linear differential equations for a spherically symmetric metric which is filled with the perfect fluid with pressure and energy density. Recently TOV equations and dynamics of neutron stars have been investigated for different types of modified gravity models from $f(R), f(G)$ and $f(T)$ ( $T$ is torsion ) numerically and also in a non-perturbative scheme [53]- [68].

The compact astrophysical objects such as black holes [69], wormholes [70],[71] and compact stars have been extensively studied withtin the framework of teleparallel gravity [72]-[81]. In fact compact stars have been theoritically modelled within the framework of $f(T)$ gravity. Their anisotropic behavior, regularity conditions, stability and surface redshift have been thorughly investigated. In this work we shall attempt to model anisotropic compact stars as fluid spheres within the framework of $f(T)$ gravity. We have analysed our model for anisotropic behaviour, stablity and surface redshift.

Our plan in this paper is the following scheme: In Sec. (II) we present $f(T)$ theory as an alternative theory for gravity. In Sec. (III) we derive equations of motion for a spherically symmetric star. In Sec. (IV) we study an isotropic model of compact star using astrophysical data. We conclude and summarize in Sec. (V). Some preliminary formulae are presented in Sec. (VI).

\section{THE TELEPARALLEL EQUIVALENT OF GENERAL RELATIVITY}

To begin with $f(T)$ theory, to keep away from any perplexity, let us characterize the notion of the latin subscript $i, j$ as those related to the tetrad field $\theta_{\mu}^{i}$ and greek $\mu, \nu$ one identified with the space time coordinates. The line element of the manifold is given by

$$
d s^{2}=g_{\mu \nu} d x^{\mu} d x^{\nu}
$$

The above metric can be rewritten by the tetrads basis, defined by

$$
\begin{gathered}
d S^{2}=g_{\mu \nu} d x^{\mu} d x^{\nu}=\eta_{i j} \theta_{\mu}^{i} \theta_{\nu}^{j} d x^{\mu} d x^{\nu}, \\
d x^{\mu}=e_{i}^{\mu} \theta^{i}, \theta^{i}=e_{\mu}^{i} d x^{\mu},
\end{gathered}
$$

where $\eta_{i j}=\operatorname{diag}[1,-1,-1,-1]$ and $e_{i}^{\mu} e_{i}^{\nu}=\delta_{\nu}^{\mu}$ or $e_{i}^{\mu} e_{j}^{\nu}=\delta_{i}^{j}$.

The root of the metric determinant is given by $\sqrt{-g}=\operatorname{det}\left[e_{\mu}^{i}\right]=e$. The Weitzenböck's anti-symmetric connection components in Weitzenböck's spacetime [80] for vanishing Riemann tensor part and non-vanishing torsion term are defined as

$$
\Gamma_{\mu \nu}^{\alpha}=e_{i}^{\alpha} \partial_{\nu} e_{\mu}^{i}=-e_{i}^{\mu} \partial_{\nu} e_{i}^{\alpha}
$$

The torsion and the contorsion are defined by

$$
\begin{gathered}
T_{\mu \nu}^{\alpha}=\Gamma_{\nu \mu}^{\alpha}-\Gamma_{\mu \nu}^{\alpha}=e_{i}^{\alpha}\left(\partial_{\mu} e_{\nu}^{i}-\partial_{\nu} e_{\mu}^{i}\right) \\
K_{\alpha}^{\mu \nu}=-\frac{1}{2}\left(T_{\alpha}^{\mu \nu}-T_{\alpha}^{\nu \mu}-T_{\alpha}^{\mu \nu}\right)
\end{gathered}
$$

and the components of the tensor $S_{\alpha}^{\mu \nu}$ as

$$
S_{\alpha}^{\mu \nu}=\frac{1}{2}\left(K_{\alpha}^{\mu \nu}+\delta_{\alpha}^{\mu} T_{\beta}^{\beta \nu}-\delta_{\alpha}^{\nu} T_{\beta}^{\beta \mu}\right)
$$

Here, torsion scalar is

$$
T=T_{\mu \nu}^{\alpha} S_{\alpha}^{\mu \nu}
$$


Analogous to $f(R)$ gravity, the action for $f(T)$ gravity is

$$
S\left[e_{\mu}^{i}, \Phi_{A}\right]=\int d^{4} x e\left[\frac{1}{16 \pi} f(T)+\mathcal{L}_{\text {matter }}\left(\Phi_{A}\right)\right],
$$

in the above action $G=c=1$ have been used and the $\mathcal{L}_{\text {matter }}\left(\Phi_{A}\right)$ is matter field. The variation of the above action providevthe following field equations in $f(T)$ gravity,

$$
S_{\mu}^{\nu \rho} \partial_{\rho} T f_{T T}+\left[e^{-1} e_{\mu}^{i} \partial_{\rho}\left(e e_{i}^{\alpha} S_{\alpha}^{\nu \rho}\right)+T_{\lambda \mu}^{\alpha} S_{\alpha}^{\nu \lambda}\right] f_{T}+\frac{1}{4} \delta_{\mu}^{\nu} f=4 \pi \mathcal{T}_{\mu}^{\nu},
$$

where $\mathcal{T}_{\mu}^{\nu}$ is matter. In the present case, we take the matter as anisotropic fluid for which energy-momentum tensor is

$$
\mathcal{T}_{\mu}^{\nu}=\left(\rho+p_{t}\right) u_{\mu} u^{\nu}-p_{t} \delta_{\mu}^{\nu}+\left(p_{r}-p_{t}\right) v_{\mu} v^{\nu}
$$

where $u^{\mu}$ and $v^{\mu}$ are the four-velocity and radial-four vectors, respectively. Further, $p_{r}$ and $p_{t}$ are pressures along radial and tansverse directions.

\section{MODEL OF ANISOTROPIC COMPACT STARS IN GENERALIZED TELLEPARALLEL GRAVITY}

We assume the geometry of star in the form of static spherically symmetric spacetime is given

$$
d s^{2}=e^{a(r)} d t^{2}-e^{b(r)} d r^{2}-r^{2}\left(d \theta^{2}+\sin ^{2}(\theta) d \phi^{2}\right) .
$$

We introduce the tetrad matrix for (12) as follows:

$$
\left[e_{\mu}^{i}\right]=\operatorname{diag}\left[e^{\frac{a(r)}{2}}, e^{\frac{b(r)}{2}}, r, r \sin (\theta)\right]
$$

One can obtain $e=\operatorname{det}\left[e_{\mu}^{i}\right]=e^{\frac{(a+b)}{2}} r^{2} \sin (\theta)$, and we determine the torsion scalar and its derivative in terms of $r$ as

$$
\begin{aligned}
T(r) & =\frac{2 e^{-b}}{r}\left(a^{\prime}+\frac{1}{r}\right), \\
T^{\prime}(r) & =\frac{2 e^{-b}}{r}\left(a^{\prime \prime}+\frac{1}{r^{2}}-T\left(b^{\prime}+\frac{1}{r}\right)\right),
\end{aligned}
$$

where the prime denotes the derivative with respect to $r$. The set of equations for an anisotropic fluid as [70],[71]

$$
\begin{aligned}
4 \pi \rho= & \frac{f}{4}-\frac{f_{T}}{2}\left(T-\frac{1}{r^{2}}-\frac{e^{-b}}{r}\left(a^{\prime}+b^{\prime}\right)\right), \\
4 \pi p_{r}= & \frac{f_{T}}{2}\left(T-\frac{1}{r^{2}}\right)-\frac{f}{4}, \\
4 \pi p_{t}= & {\left[\frac{T}{2}+e^{-b}\left(\frac{a^{\prime \prime}}{2}+\left(\frac{a^{\prime}}{4}+\frac{1}{2 r}\right)\left(a^{\prime}-b^{\prime}\right)\right)\right] \frac{f_{T}}{2}-\frac{f}{4}, } \\
& \frac{\cot \theta}{2 r^{2}} T^{\prime} f_{T T}=0 .
\end{aligned}
$$

Further, the last equation leads to the the following linear form of $f(T)$ :

$$
f(T)=\beta T+\beta_{1}
$$

where $\beta$ and $\beta_{1}$ are integration constants. We parameterize the metric as the following:

$$
e^{a(r)}=\left(1+b r^{2}\right)^{2}, e^{b(r)}=\left(A+\frac{B\left(2+b r^{2}\right)^{3 / 2}}{3 \sqrt{b}}\right)^{2}
$$

where $b$ is a constant, the arbitrary constants $A, B$ and $b$ can be evaluated by using some physical matching conditions. Now using equations given in (16-19), we will get forms of matter components given in equations (38-43) in appendix Sec. (VI).

The behavior of density, radial and transverse pressures and equation of state (EoS) parameters are given in figures 1-5 for a strange star candidate RX J 1856-37, Her X-1, and Vela X-12, respectively. In this case EoS parameters have values $\omega_{t}>0$ and $0<\omega_{r} \leq 1$, which shows the fact that star consists of ordinary matter and effect of $f(T)$ model in the present setup. 

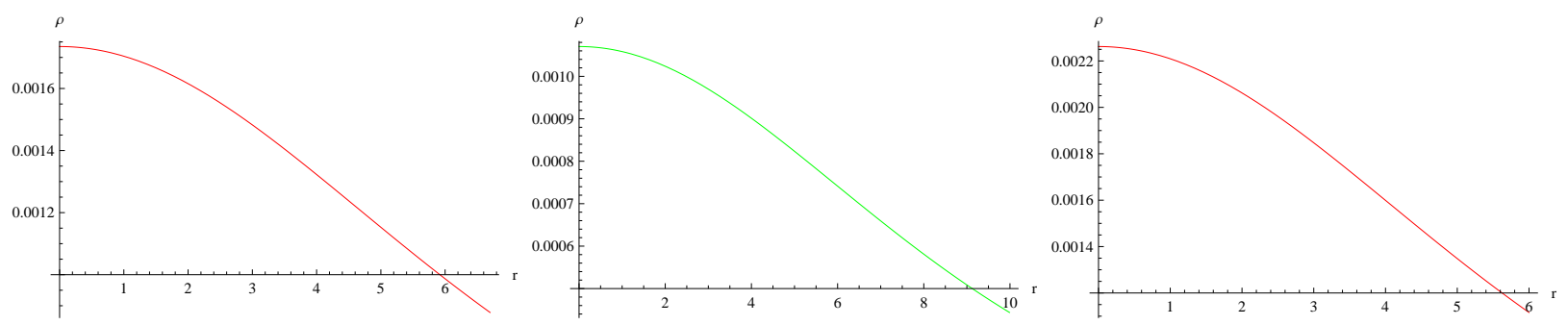

Figure 1: Density variation for Strange star candidate RX J 1856-37, Her X-1, and Vela X-12, respectively.
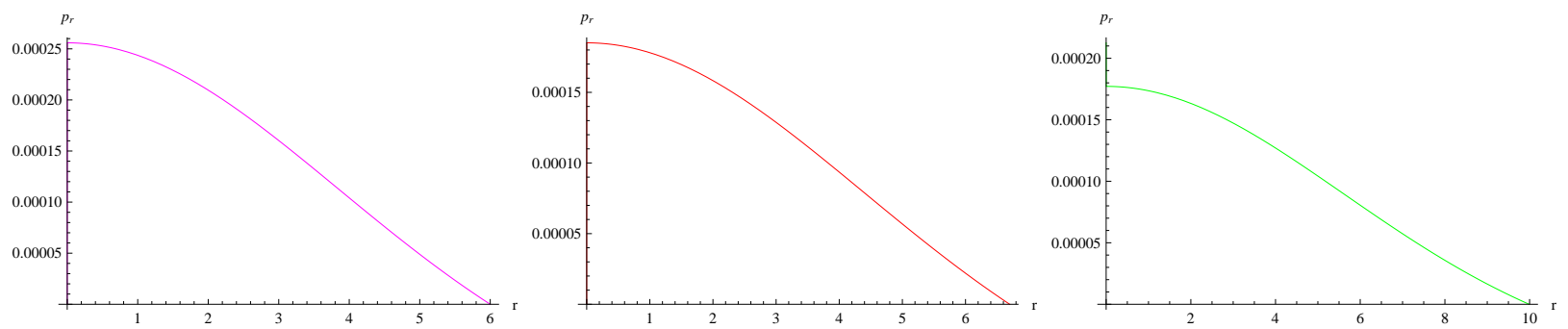

Figure 2: Radial pressure variation for Strange star candidate RX J 1856-37, Her X-1, and Vela X-12, respectively.
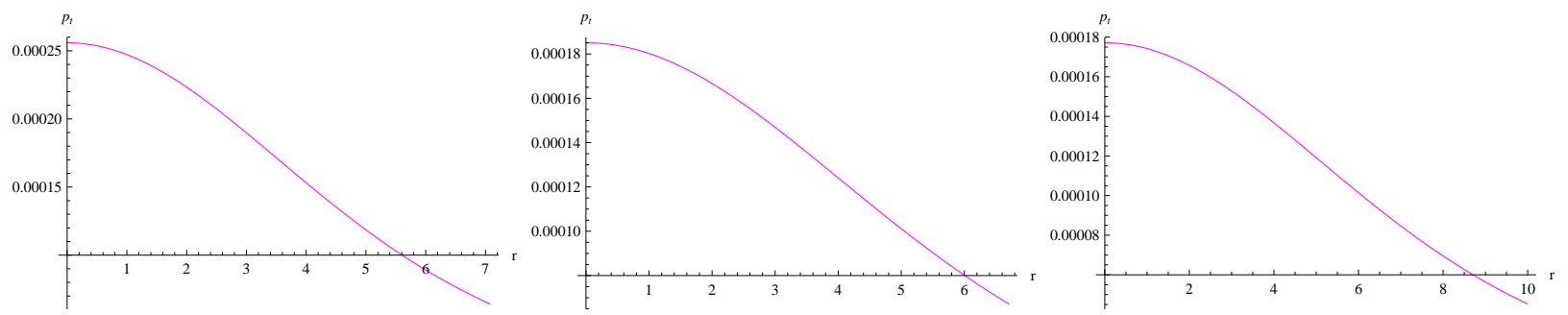

Figure 3: Transverse pressure variation for Strange star candidate RX J 1856-37, Her X-1, and Vela X-12, respectively.

Figure 4: EOS parameter $\omega_{r}$ variation for Strange star candidate RX J 1856-37, Her X-1, and Vela X-12, respectively.
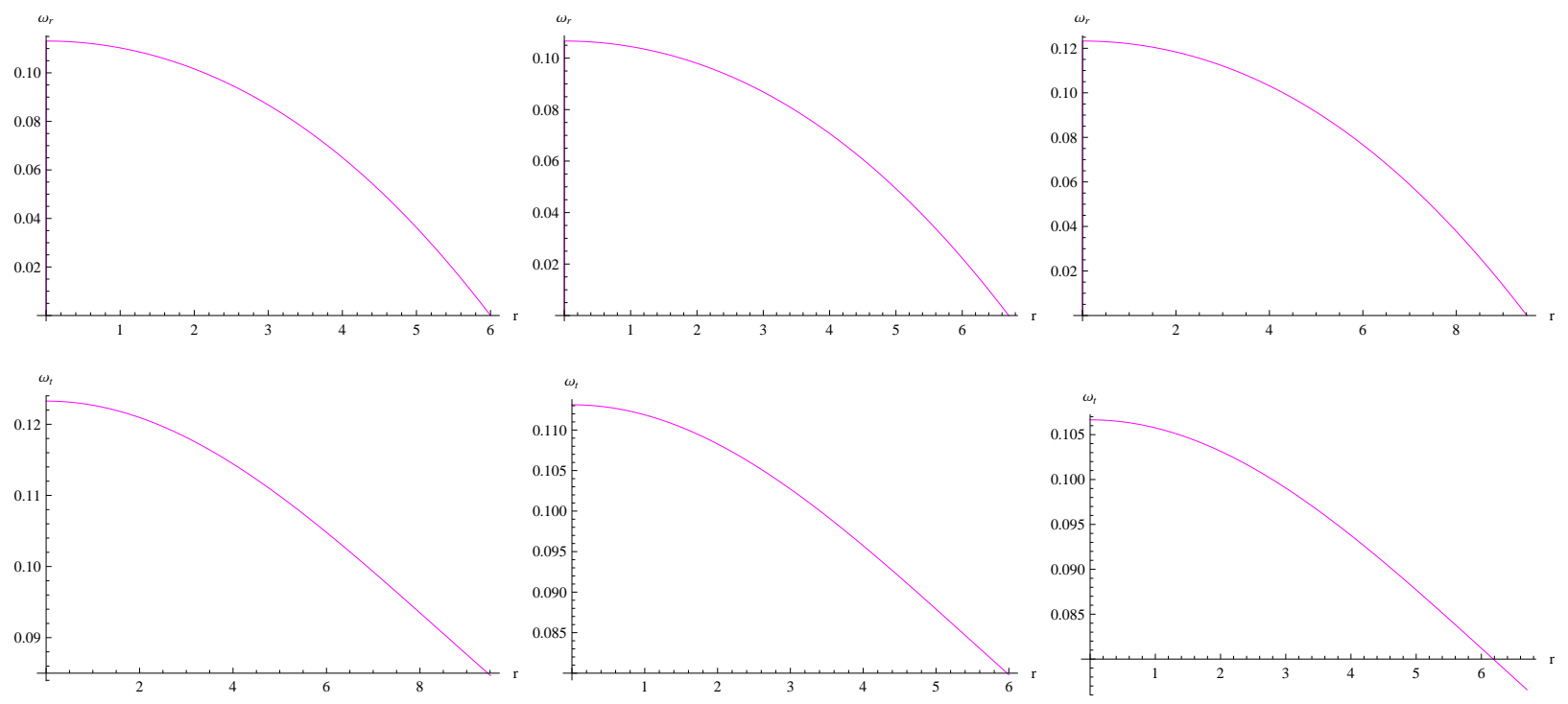

Figure 5: EOS parameter $\omega_{t}$ variation of Strange star candidate Cen-X-3, RX J 1856-37 and Her X-1, respectively. 


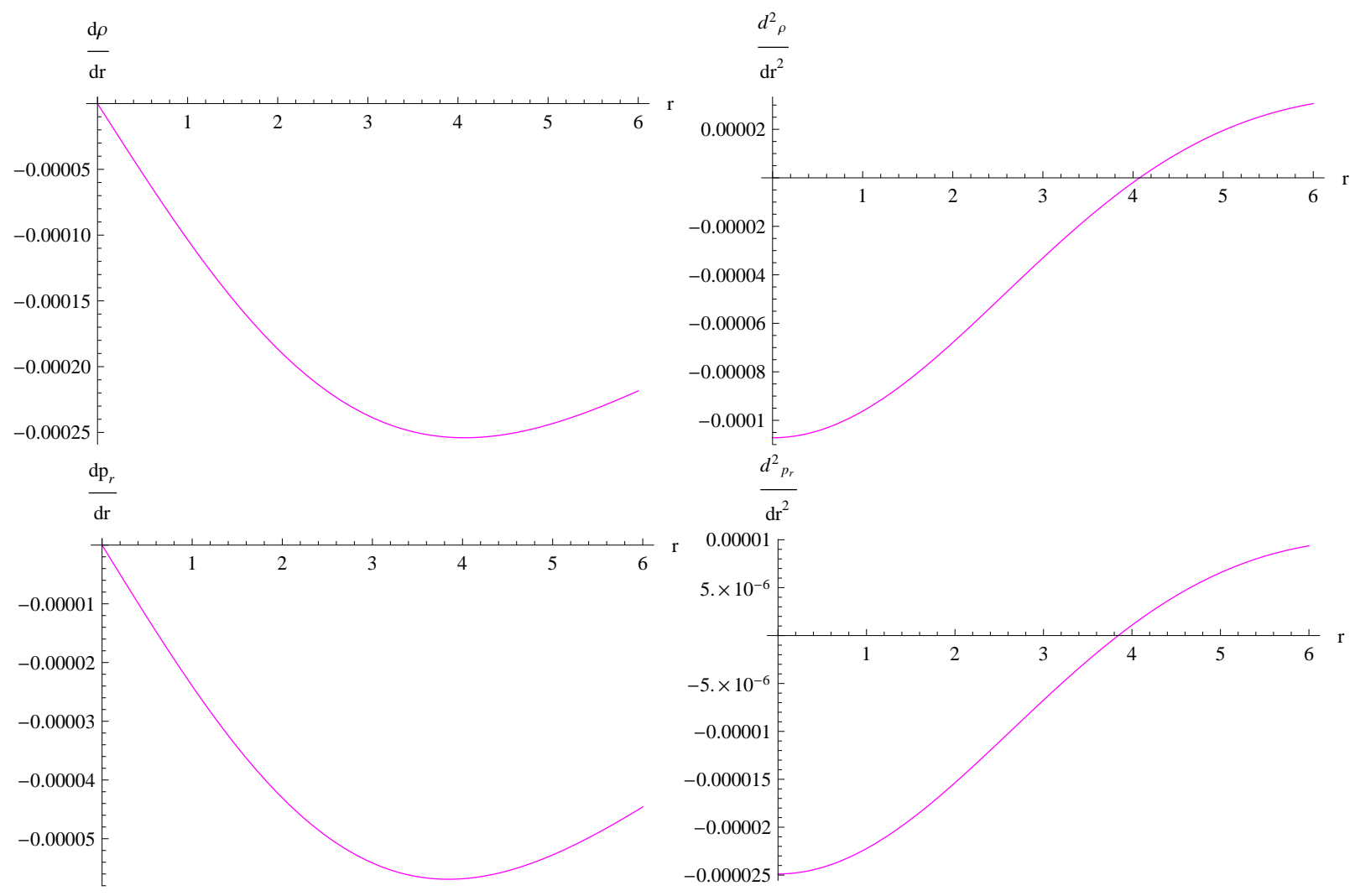

Figure 6: The plotted graphs are only for the data of $4 \mathrm{U}$ 1820-30.

\section{ANALYSIS OF THE PROPOSED MODEL}

Here, we discuss the following properties of the proposed model:

\section{A. Anisotropic Behavior}

From Eqs.(38-43), we get the radial gradient of pressures . We compute second order derivatives $\frac{d^{2} \rho}{d r^{2}}$. We observe that at center $r=0$, our model provides that

$$
\begin{aligned}
& \frac{d \rho}{d r}=0, \quad \frac{d p_{r}}{d r}=0 \\
& \frac{d^{2} \rho}{d r^{2}}<0, \quad \frac{d^{2} p_{r}}{d r^{2}}<0 .
\end{aligned}
$$

This indicate maximality of radial pressure and density. This fact implies that $\rho$ and $p_{r}$ are decreasing function of $r$ as shown in figures $\mathbf{1 , 2}$ for a class of strange stars.

The measure of anisotropy is

$$
\Delta=\frac{2}{r}\left(p_{t}-p_{r}\right)
$$

which takes the form given in Eq. (44).

It is well known that anisotropy will be directed outward when $p_{t}>p_{r}$ i.e., $\Delta>0$, and inward when $p_{t}<p_{r}$ i.e., $\Delta<0$. It is apparent from the figure $\mathbf{7}$ that for our model that a repulsive (anisotropic) force would exists as $(\Delta>0)$ (for smaller values of $r$ ) which permits the formation of super massive star, while for larger values of $r, \Delta=0$, where a star comes to the equilibrium position.

The anisotropy will be directed outward when $p_{t}>p_{r}$ this implies that $\Delta>0$ and directed inward when $p_{t}<p_{r}$ implying $\Delta>0$. In this case $\Delta>0$, for larger values of $r$ for a class of strange stars as shown in figures 7 . This implies that anisotropic force allows the construction of more massive stars . 

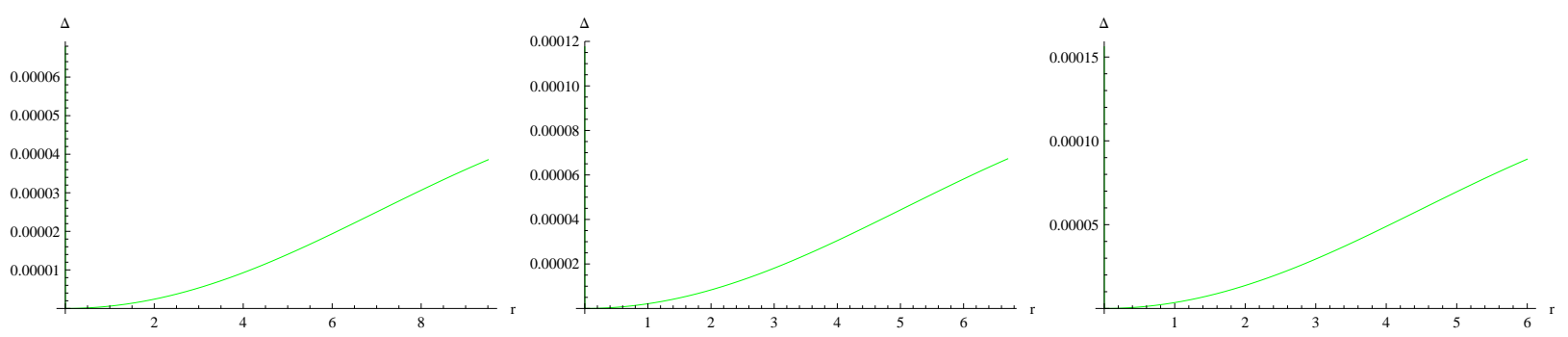

Figure 7: Variation of anisotropy $\Delta$ for Strange star candidate Her X-1, SAX J 1808.4-3658(SS1) and 4U 1820-30, respectively.

In order to comprehend some general results associated with the strong gravitational fields, we include weakvenergy condition (WEC), null energy condition (NEC), strong energy condition (SEC) and dominant energy condition (DEC). For an anisotropic fluid, these are defined as

$$
\begin{aligned}
\text { NEC : } & \rho+p_{r} \geq 0, \quad \rho+p_{t} \geq 0, \\
\text { WEC : } & \rho \geq 0, \quad \rho+p_{r} \geq 0, \quad \rho+p_{t} \geq 0, \\
\text { SEC : } & \rho+p_{r} \geq 0, \quad \rho+p_{t} \geq 0, \quad \rho+p_{r}+2 p_{t} \geq 0, \\
\text { DEC : } & \rho>\left|p_{r}\right|, \quad \rho>\left|p_{t}\right| .
\end{aligned}
$$

We find that our model satisfies these conditions .

\section{B. Matching of interior and exteriorvspacetime}

The intrinsic metric of the boundary surface will be the same whether it is derived from the internal or external geometry of the star. It proves that for any well defined coordinate system, the metric tensor components will be continuous across the boundary surface. for this purpose the matching conditions are equested for the interior metric . In this case, one can solve the system of field equations with auxiliary boundary condition $p_{r}(r=R)=0$. It is needed to match smoothly the interior metric to an exterior Schwarzschild metric given by

$$
d s^{2}=\left(1-\frac{2 M}{r}\right) d t^{2}-\left(1-\frac{2 M}{r}\right)^{-1} d r^{2}-r^{2}\left(d \theta^{2}+\sin ^{2} \theta d \varphi^{2}\right) .
$$

At the surface of star, $r=R$ continuity of the metric functions $g_{t t}, g_{r r}$ and $\frac{\partial g_{t t}}{\partial r}$ at the boundary surface yield,

$$
g_{t t}^{-}=g_{t t}^{+}, \quad g_{r r}^{-}=g_{r r}^{+}, \quad \frac{\partial g_{t t}^{-}}{\partial r}=\frac{\partial g_{t t}^{+}}{\partial r},
$$

where - and + , correspond to interior and exterior solutions. By matching the interior solution (12) and exterior solution (??) at the boundary surface $r=r_{c}$, we obtain following three equations

$$
\begin{aligned}
e^{a_{c}} & =1-\frac{2 M}{r_{c}}=\left(A+\frac{B\left(2+b r^{2}\right)^{3 / 2}}{3 \sqrt{b}}\right)^{2}, \\
e^{-b_{c}} & =1-\frac{2 M}{r_{c}}=\left(1+b r_{c}^{2}\right)^{-2} \\
p_{r}\left(r_{c}\right) & =0
\end{aligned}
$$

Using the boundary condition $(26-28)$ we obtain

$$
\frac{A}{B}=\frac{2-4 r_{c}^{2}-b^{2} r_{c}^{4}}{3 \sqrt{b} \sqrt{2+b r_{c}^{2}}}
$$




$$
\begin{gathered}
A=\left(1+b r_{c}^{2}\right)^{-1}-\frac{B\left(2+b r_{c}^{2}\right)^{3 / 2}}{3 \sqrt{b}} \\
\frac{2 M}{r_{c}}=1-\left(1+b r_{c}^{2}\right)^{-2}
\end{gathered}
$$

Solving $(29-31)$ we obtain,

$$
\begin{gathered}
B=\frac{\sqrt{b}}{2} \frac{\sqrt{2+b r_{c}^{2}}}{1+b r_{c}^{2}} \\
A=\frac{2-2-4 r_{c}^{2}-b^{2} r_{c}^{4}}{6\left(1+b r_{c}^{2}\right)} \\
b=\frac{1}{r_{c}^{2}}\left[\frac{1}{\sqrt{1-\frac{2 M}{r_{c}}}}-1\right]
\end{gathered}
$$

Now the values of constants $A, b$ and $B$ for some well known compact stars are obtained in table $\mathbf{1}$.

Table I: The values of constants are obtained from model for some well known model compact stars

\begin{tabular}{|c|c|c|c|c|c|c|}
\hline Compact Star & Mass & Radius $(\mathrm{km})$ & $b\left(\mathrm{~km}^{-2}\right)$ & $B\left(\mathrm{~km}^{-1}\right)$ & $A$ & $\frac{M}{r}$ \\
\hline Her X-1 & $0.98 M_{\odot}$ & 6.7 & 0.007268 & 0.049020 & 0.073950 & 0.215746 \\
\hline RX J 1856-37 & $0.9031 M_{\odot}$ & 6 & 0.009475 & 0.055529 & 0.064509 & 0.222012 \\
\hline Vela X-12 & $1.77 M_{\odot}$ & 9.99 & 0.004483 & 0.036184 & 0.001172 & 0.261336 \\
\hline Cen X-3 & $1.49 M_{\odot}$ & 9.51 & 0.004020 & 0.035744 & 0.050524 & 0.231098 \\
\hline
\end{tabular}

For the given values of $M$ and $R$ for given star, the constants $A$ and $B$ are given in the table $\mathbf{1}$. We would like to mention that the values of $M$ and $R$ have been taken from [82], [83], [84] to calculate the values of $A$ and $B$.

\section{Stability}

Here, we discuss the stability of strange stars for this purpose the radial sound speed $v_{s r}$ and transverse sound speed $v_{s t}^{2}$ are given by

$$
\begin{aligned}
& v_{s r}^{2}=\frac{d p_{r}}{d \rho} \equiv \frac{-\left(3\left(\begin{array}{c}
108 A^{3} b^{3 / 2}\left(2+b r^{2}\right)^{3 / 2}+9 A^{2} b B\left(1+b r^{2}\right)\left(56+b r^{2}\left(47+9 b r^{2}\right)\right. \\
-2 B^{3}\left(2+b r^{2}\right)^{3}\left(10+b r^{2}\left(7+b r^{2}\left(2+b r^{2}\right)\right)\right) \\
+3 A \sqrt{b} B^{2}\left(2+b r^{2}\right)^{3 / 2}\left(4+b r^{2}\left(41+b r^{2}\left(28+3 b r^{2}\right)\right)\right)
\end{array}\right)\right)}{2\left(2+b r^{2}\right)\left(3 A \sqrt{b}+B\left(2+b r^{2}\right)^{3 / 2}\right)} \\
& \times\left(\begin{array}{c}
9 A^{2} b \sqrt{2+b r^{2}}\left(12+\left(1+b r^{2}\right)\left(3+b r^{2}\right) \beta\right) \\
+B^{2}\left(2+b r^{2}\right)^{3 / 2} \\
96-20 \beta+ \\
\left(78-18 \beta+b r^{2}\left(\begin{array}{c} 
\\
b r^{2}\left(30+\left(5+b r^{2}\left(8+b r^{2}\right)\right) \beta\right)
\end{array}\right)\right) \\
126+26 \beta \\
+3 A \sqrt{b} B\left(3(39+\beta)+b r^{2}\left(\begin{array}{c}
1 \\
+b r^{2}\left(33+\left(37+2 b r^{2}\left(8+b r^{2}\right)\right) \beta\right)
\end{array}\right)\right)
\end{array}\right)
\end{aligned}
$$



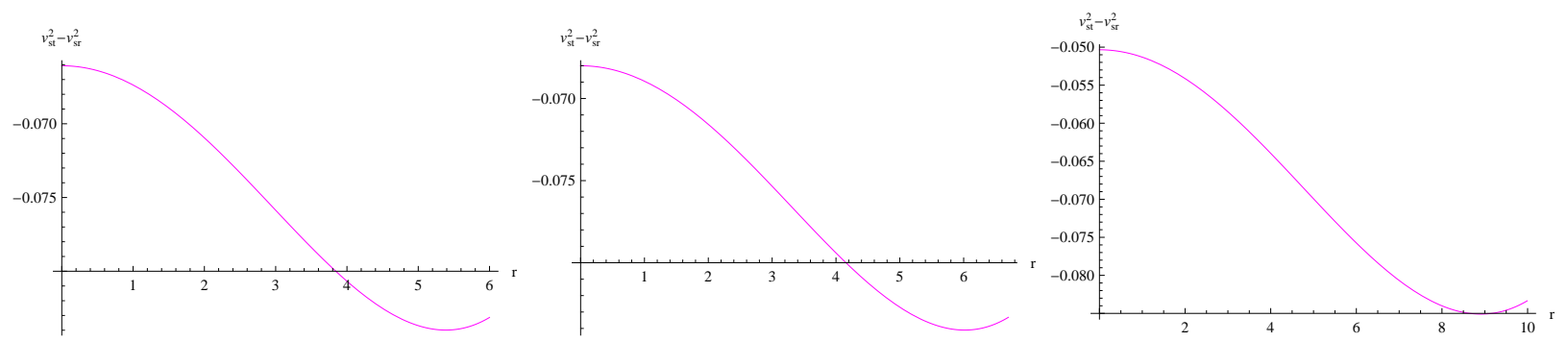

Figure 8: Variation of $v_{s t}^{2}-v_{s r}^{2}$ for Strange star candidate Her X-1, SAX J 1808.4-3658(SS1) and 4U 1820-30, respectively.
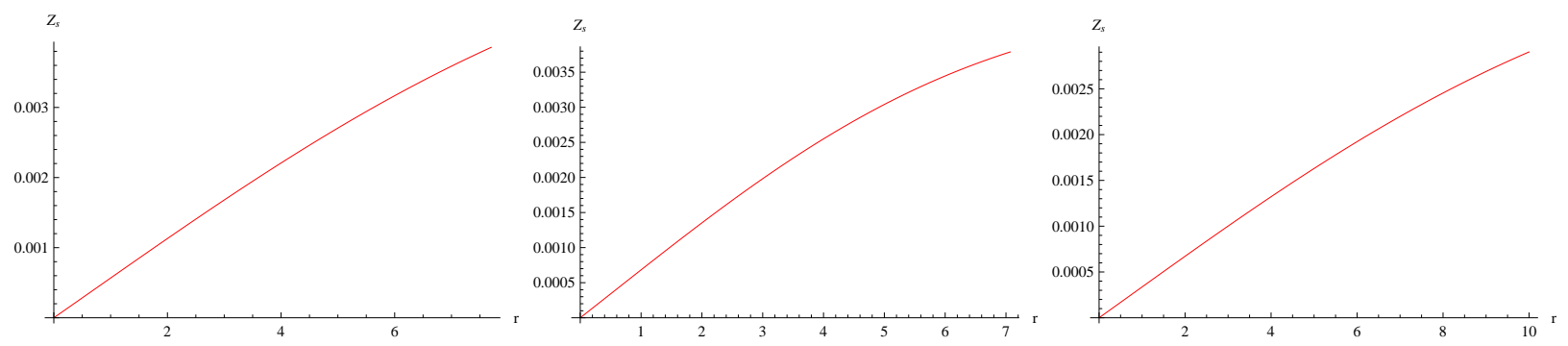

Figure 9: Variation of surface redshift $Z_{s}$ for Strange star candidate Her X-1, SAX J 1808.4-3658(SS1) and 4U 1820-30, respectively.

$$
\begin{aligned}
& v_{s t}^{2}=\frac{d p_{t}}{d \rho} \\
& \equiv-\frac{\left(1+b r^{2}\right)\left(9 A^{2} b \sqrt{2+b r^{2}}\left(3+b r^{2}\right)+B^{2}\left(2+b r^{2}\right)^{3 / 2}\left(-18+b r^{2}\left(-2+b r^{2}\left(7+b r^{2}\right)\right)\right)\right) \beta}{9 A^{2} b \sqrt{2+b r^{2}}\left(12+\left(1+b r^{2}\right)\left(3+b r^{2}\right) \beta\right)} \\
& \left(1+b r^{2}\right)\left(3 A \sqrt{b} B\left(3+b r^{2}\left(23+2 b r^{2}\left(7+b r^{2}\right)\right)\right)\right) \beta \\
& \left.\begin{array}{c}
9 A^{2} b \sqrt{2+b r^{2}}\left(12+\left(1+b r^{2}\right)\left(3+b r^{2}\right) \beta\right) \\
+B^{2}\left(2+b r^{2}\right)^{3 / 2}\left(78-18 \beta+b r^{2}\left(96-20 \beta+b r^{2}\left(30+\left(5+b r^{2}\left(8+b r^{2}\right)\right) \beta\right)\right)\right) \\
+3 A \sqrt{b} B\left(3(39+\beta)+b r^{2}\left(126+26 \beta+b r^{2}\left(33+\left(37+2 b r^{2}\left(8+b r^{2}\right)\right) \beta\right)\right)\right)
\end{array}\right)
\end{aligned}
$$

Reference [85] developed a proposal to check the stability of anisotropic gravitating source. Currently, this technique is termed as cracking concept which states that if radial speed of sound is greater than the transverse speed of sound in a region then such a region is a potentially stable region, otherwise unstable region. In our case, figure 8 indicates that there is change of sign for the term $v_{s t}^{2}-v_{s r}^{2}$ within the specific configuration. We can see that $\left|v_{s t}^{2}-v_{s r}^{2}\right| \leq 1$. This is used to check whether local anisotropic matter distribution is stable or not. Hence, we conclude that our strange star model is unstable.

\section{Surface Redshift}

The compactness of the star is given by

$$
u \equiv e^{-a}=1-\frac{2 m}{r}
$$

In our model the effective mass is given by 


$$
m(r)=4 \pi \int_{0}^{r} \rho r^{2} d r
$$

The surface redshift $\left(Z_{s}\right)$ resulting from the compactness $u$ is obtained as

$$
1+Z_{s}=[1-2 u]^{\frac{-1}{2}}
$$

where

$$
1+Z_{s}=\left[1-\left(\frac{2 b \sqrt{A} \pi \beta+\sqrt{\pi} \beta\left(\pi-2 A \pi r^{2}\right) \operatorname{erf}(\sqrt{A} b)+b \pi r^{2} \beta_{1} \sqrt{A}}{4 \pi \sqrt{A}}\right)\right]^{\frac{-1}{2}} .
$$

The maximum value of the surface redshift for the compact stars is shown in figure $\mathbf{9}$.

\section{DISCUSSION AND CONCLUSION}

Different cosmological observations have been shown that our Universe undergoes two phases of accelerated expansion, early inflationary era and late time acceleration. Dark energy in the form of exotic fluid or modified gravity or cosmological constant term are widely studied for this purpose. Numerous forms of modified gravity proposed and one recently proposal is to use torsion in a modified theory, called as $f(T)$ gravity, is one of the most popular modifications of General Relativity.

In this article, authors demonstrated analytical models of compact stars under the framework of $f(T)$ gravity with the anisotropic gravitating static source. Linear form of $f(T)$ gravity model is considered here, Moreover the stars are supposed to be anisotropic in their internal structure. It is observed that the analytic solution in $f(T)$ gravity have a matching by the interior metric with the well-known exterior metric. The following properties show the physical analysis of the obtained results regarding the anisotropic compact stars in $f(T)$ gravity:

- EoS parameters bound are given by $\omega_{t}>0$ and $\omega_{r}>0$, that is steady with ordinary matter distribution in $f(T)$ gravity.

- The density and pressures both are decreasing functions that attain the maximum value at the center.

- It has been shown that the anisotropy will be directed outward when $p_{t}>p_{r}$ this means that $\Delta>0$ and directed inward when $p_{t}<p_{r}$ implying $\Delta<0$. For larger values of $r$, of different strange stars in our case $\Delta>0$, which tells that anisotropic force is most beneficial for construction of more massive stars in $f(T)$ gravity.

- Variation of $v_{s t}^{2}-v_{s r}^{2}$ for different strange stars shows that $\left|v_{s t}^{2}-v_{s r}^{2}\right| \leq 1$. In this way, our suggested strange star model shows more stablity in $f(T)$ gravity.

- In this paper, we have used the diagonal tetrad to discuss the dynamics of the gravitating source. Also, one can use the off diagonal tetrad to study the compact stars.

\section{APPENDIX}

In this appendix we present exact forms of different quatities which have been used in our analysis.

$$
\begin{aligned}
& \rho=\frac{b\left(3 A \sqrt{b}\left(4+\left(2+3 b r^{2}+b^{2} r^{4}\right) \beta\right)\right)}{8 \pi\left(1+b r^{2}\right)^{3}\left(3 A \sqrt{b}+B\left(2+b r^{2}\right)^{3 / 2}\right)} \\
& +\frac{B \sqrt{2+b r^{2}}\left(-2(-7+\beta)+5 b^{2} r^{4} \beta+b^{3} r^{6} \beta+2 b r^{2}(5+\beta)\right)}{8 \pi\left(1+b r^{2}\right)^{3}\left(3 A \sqrt{b}+B\left(2+b r^{2}\right)^{3 / 2}\right)}+\frac{\beta}{8 \pi r^{2}}-\frac{\beta_{1}}{16 \pi},
\end{aligned}
$$




$$
\begin{gathered}
p_{r}=\frac{\left(3 A \sqrt{b}+B \sqrt{2+b r^{2}}\left(2+7 b r^{2}\right)\right) \beta}{8 \pi\left(r+b r^{3}\right)^{2}\left(3 A \sqrt{b}+B\left(2+b r^{2}\right)^{3 / 2}\right)}-\frac{\beta}{8 \pi r^{2}}-\frac{\beta_{1}}{16}, \\
p_{t}=\frac{b\left(-18 A^{2} b \sqrt{2+b r^{2}}+B^{2}\left(2+b r^{2}\right)^{3 / 2}\left(4+b r^{2}+b^{2} r^{4}\right)-3 A \sqrt{b} B\left(4+b r^{2}\left(7+b r^{2}\right)\right)\right)}{8 \pi\left(1+b r^{2}\right)^{3} \sqrt{2+b r^{2}}\left(3 A \sqrt{b}+B\left(2+b r^{2}\right)^{3 / 2}\right)^{2}} \\
+\frac{3 A b \sqrt{b} B\left(4+b r^{2}\left(7+b r^{2}\right)\right)}{8 \pi\left(1+b r^{2}\right)^{3} \sqrt{2+b r^{2}}\left(3 A \sqrt{b}+B\left(2+b r^{2}\right)^{3 / 2}\right)^{2}}-\frac{\beta_{1}}{16 \pi},
\end{gathered}
$$

Also, the equation of state (EOS) parameters can be written as

$$
\begin{aligned}
& \omega_{r}(r)=-\frac{\left(1+b r^{2}\right) 3 A \sqrt{b}\left(2+b r^{2}\right) \beta}{3 A \sqrt{b}\left(4+\left(2+3 b r^{2}+b^{2} r^{4}\right) \beta\right)+B \sqrt{2+b r^{2}}\left(14-2 \beta+5 b^{2} r^{4} \beta+b^{3} r^{6} \beta+2 b r^{2}(5+\beta)\right)} \\
& -\frac{B\left(1+b r^{2}\right) \sqrt{2+b r^{2}}\left(-2+4 b r^{2}+b^{2} r^{4}\right) \beta}{3 A \sqrt{b}\left(4+\left(2+3 b r^{2}+b^{2} r^{4}\right) \beta\right)+B \sqrt{2+b r^{2}}\left(14-2 \beta+5 b^{2} r^{4} \beta+b^{3} r^{6} \beta+2 b r^{2}(5+\beta)\right)} \\
& \omega_{t}(r)=\frac{-18 A^{2} b \sqrt{2+b r^{2}}+B^{2}\left(2+b r^{2}\right)^{3 / 2}\left(4+b r^{2}+b^{2} r^{4}\right)}{\sqrt{2+b r^{2}}\left(3 A \sqrt{b}+B\left(2+b r^{2}\right)^{3 / 2}\right)} \\
& \times \frac{1}{\left(3 A \sqrt{b}\left(4+\left(1+b r^{2}\right)\left(2+b r^{2}\right) \beta\right)+B \sqrt{2+b r^{2}}\left(14-2 \beta+b r^{2}\left(10+\left(2+b r^{2}\left(5+b r^{2}\right)\right) \beta\right)\right)\right)} \\
& -\frac{1}{\sqrt{2+b r^{2}}\left(3 A \sqrt{b}+B\left(2+b r^{2}\right)^{3 / 2}\right)} \\
& \times \frac{3 A \sqrt{b} B\left(4+b r^{2}\left(7+b r^{2}\right)\right)}{\left(3 A \sqrt{b}\left(4+\left(1+b r^{2}\right)\left(2+b r^{2}\right) \beta\right)+B \sqrt{2+b r^{2}}\left(14-2 \beta+b r^{2}\left(10+\left(2+b r^{2}\left(5+b r^{2}\right)\right) \beta\right)\right)\right)} \\
& \frac{d^{2} \rho}{d r^{2}}=\frac{3 b^{2}\left(27 A^{3} b^{3 / 2}\left(2+b r^{2}\right)^{3 / 2}\left(-4-\beta+b r^{2}\left(28+\left(3+b r^{2}\left(5+b r^{2}\right)\right) \beta\right)\right)\right.}{\left(4 \pi\left(1+b r^{2}\right)^{5}\left(2+b r^{2}\right)^{3 / 2}\left(3 A \sqrt{b}+B\left(2+b r^{2}\right)^{3 / 2}\right)^{3}\right)} \\
& +\frac{B^{3} 3 b^{2}\left(2+b r^{2}\right)^{3}\left(-52+12 \beta+b r^{2}\left(250-62 \beta+b r^{2}\left(538-156 \beta+b r^{2}(338-89 \beta)\right)\right)\right)}{\left(4 \pi\left(1+b r^{2}\right)^{5}\left(2+b r^{2}\right)^{3 / 2}\left(3 A \sqrt{b}+B\left(2+b r^{2}\right)^{3 / 2}\right)^{3}\right)} \\
& +\frac{B^{3} 3 b^{2}\left(2+b r^{2}\right)^{3} b r^{2}\left(70+\left(3+b r^{2}\left(11+b r^{2}\right)\right) \beta\right)}{\left(4 \pi\left(1+b r^{2}\right)^{5}\left(2+b r^{2}\right)^{3 / 2}\left(3 A \sqrt{b}+B\left(2+b r^{2}\right)^{3 / 2}\right)^{3}\right)} \\
& +\frac{9 b^{2} A \sqrt{b} B^{2}\left(2+b r^{2}\right)^{3 / 2}\left(10(-13+\beta)+b r^{2}\left(622-58 \beta+b r^{2}\left(1214-68 \beta+3 b r^{2}(226+23 \beta)\right)\right)\right)}{\left(4 \pi\left(1+b r^{2}\right)^{5}\left(2+b r^{2}\right)^{3 / 2}\left(3 A \sqrt{b}+B\left(2+b r^{2}\right)^{3 / 2}\right)^{3}\right)}
\end{aligned}
$$




$$
\begin{aligned}
& +\frac{9 b^{2} A \sqrt{b} B^{2}\left(2+b r^{2}\right)^{3 / 2} b r^{2}\left(40+\left(33+b r^{2}\left(11+b r^{2}\right)\right) \beta\right)}{\left(4 \pi\left(1+b r^{2}\right)^{5}\left(2+b r^{2}\right)^{3 / 2}\left(3 A \sqrt{b}+B\left(2+b r^{2}\right)^{3 / 2}\right)^{3}\right)} \\
& +\frac{27 A^{2} B b^{3}\left(-10(11+\beta)+b r^{2}\left(566-2 \beta+b r^{2}\left(2(521+52 \beta)+3 b r^{2}(186+63 \beta)\right)\right)\right)}{\left(4 \pi\left(1+b r^{2}\right)^{5}\left(2+b r^{2}\right)^{3 / 2}\left(3 A \sqrt{b}+B\left(2+b r^{2}\right)^{3 / 2}\right)^{3}\right)} \\
& +\frac{27 A^{2} B b^{4} r^{2}\left(32+\left(41+b r^{2}\left(11+b r^{2}\right)\right) \beta\right)}{\left(4 \pi\left(1+b r^{2}\right)^{5}\left(2+b r^{2}\right)^{3 / 2}\left(3 A \sqrt{b}+B\left(2+b r^{2}\right)^{3 / 2}\right)^{3}\right)} \\
& \frac{d^{2} p_{r}}{d r^{2}}=-\frac{3 b^{2}\left(27 A^{3} b^{3 / 2}\left(2+b r^{2}\right)^{3 / 2}\left(-1+b r^{2}\left(4+b r^{2}\right)\right) \beta\right)}{\left(4 \pi\left(1+b r^{2}\right)^{4}\left(2+b r^{2}\right)^{3 / 2}\left(3 A \sqrt{b}+B\left(2+b r^{2}\right)^{3 / 2}\right)^{3}\right)} \\
& -\frac{3 b^{2}\left(3 A \sqrt{b} B^{2}\left(2+b r^{2}\right)^{3 / 2}\left(10+b r^{2}\left(-68+3 b^{2} r^{4}\left(23+b r^{2}\left(10+b r^{2}\right)\right)\right)\right) \beta\right)}{\left(4 \pi\left(1+b r^{2}\right)^{4}\left(2+b r^{2}\right)^{3 / 2}\left(3 A \sqrt{b}+B\left(2+b r^{2}\right)^{3 / 2}\right)^{3}\right)} \\
& -\frac{3 b^{2}\left(B^{3}\left(2+b r^{2}\right)^{3}\left(12+b r^{2}\left(-74+b r^{2}\left(-82+b r^{2}\left(-7+b r^{2}\left(10+b r^{2}\right)\right)\right)\right) \beta\right)\right.}{\left(4 \pi\left(1+b r^{2}\right)^{4}\left(2+b r^{2}\right)^{3 / 2}\left(3 A \sqrt{b}+B\left(2+b r^{2}\right)^{3 / 2}\right)^{3}\right)} \\
& -\frac{3 b^{2}\left(9 A^{2} b B\left(-10+b r^{2}\left(8+3 b r^{2}\left(32+b r^{2}\left(31+b r^{2}\left(10+b r^{2}\right)\right)\right)\right)\right) \beta\right)}{\left(4 \pi\left(1+b r^{2}\right)^{4}\left(2+b r^{2}\right)^{3 / 2}\left(3 A \sqrt{b}+B\left(2+b r^{2}\right)^{3 / 2}\right)^{3}\right)} \\
& \Delta=\frac{b\left(-18 A^{2} b \sqrt{2+b r^{2}}+B^{2}\left(2+b r^{2}\right)^{3 / 2}\left(4+b r^{2}+b^{2} r^{4}\right)-3 A \sqrt{b} B\left(4+b r^{2}\left(7+b r^{2}\right)\right)\right)}{8 \pi\left(1+b r^{2}\right)^{3} \sqrt{2+b r^{2}}\left(3 A \sqrt{b}+B\left(2+b r^{2}\right)^{3 / 2}\right)^{2}} \\
& -\frac{\left(3 A \sqrt{b}+B \sqrt{2+b r^{2}}\left(2+7 b r^{2}\right)\right) \beta}{8 \pi\left(r+b r^{3}\right)^{2}\left(3 A \sqrt{b}+B\left(2+b r^{2}\right)^{3 / 2}\right)} \\
& \frac{d \rho}{d r}=-\frac{b^{2} r\left(9 A^{2} b \sqrt{2+b r^{2}}\left(12+\left(1+b r^{2}\right)\left(3+b r^{2}\right) \beta\right)\right)}{\left(4 \pi\left(1+b r^{2}\right)^{4} \sqrt{2+b r^{2}}\left(3 A \sqrt{b}+B\left(2+b r^{2}\right)^{3 / 2}\right)^{2}\right)} \\
& -\frac{b^{2} r\left(B^{2}\left(2+b r^{2}\right)^{3 / 2}\left(78-18 \beta+b r^{2}\left(96-20 \beta+b r^{2}\left(30+\left(5+b r^{2}\left(8+b r^{2}\right)\right) \beta\right)\right)\right)\right)}{\left(4 \pi\left(1+b r^{2}\right)^{4} \sqrt{2+b r^{2}}\left(3 A \sqrt{b}+B\left(2+b r^{2}\right)^{3 / 2}\right)^{2}\right)} \\
& -\frac{3 A \sqrt{b} B\left(3(39+\beta)+b r^{2}\left(126+26 \beta+b r^{2}\left(33+\left(37+2 b r^{2}\left(8+b r^{2}\right)\right) \beta\right)\right)\right)}{\left(4 \pi\left(1+b r^{2}\right)^{4} \sqrt{2+b r^{2}}\left(3 A \sqrt{b}+B\left(2+b r^{2}\right)^{3 / 2}\right)^{2}\right)}
\end{aligned}
$$




$$
\begin{gathered}
\frac{d p_{r}}{d r}=\frac{b^{2} r\left(9 A^{2} b \sqrt{2+b r^{2}}\left(3+b r^{2}\right)+B^{2}\left(2+b r^{2}\right)^{3 / 2}\left(-18+b r^{2}\left(-2+b r^{2}\left(7+b r^{2}\right)\right)\right)\right) \beta}{4 \pi\left(1+b r^{2}\right)^{3} \sqrt{2+b r^{2}}\left(3 A \sqrt{b}+B\left(2+b r^{2}\right)^{3 / 2}\right)^{2}} \\
+\frac{3 A \sqrt{b} B\left(3+b r^{2}\left(23+2 b r^{2}\left(7+b r^{2}\right)\right)\right) \beta}{4 \pi\left(1+b r^{2}\right)^{3} \sqrt{2+b r^{2}}\left(3 A \sqrt{b}+B\left(2+b r^{2}\right)^{3 / 2}\right)^{2}}
\end{gathered}
$$

[1] M. Sami, Curr. Sci. 97 (2009) 887 [arXiv:0904.3445 [hep-th]].

[2] M. Sami, arXiv:0901.0756 [hep-th].

[3] M. Sami, Lect. Notes Phys. 720 (2007) 219.

[4] E. J. Copeland, M. Sami and S. Tsujikawa, Int. J. Mod. Phys. D 15 (2006) 1753 [hep-th/0603057].

[5] M. Sami and N. Dadhich, TSPU Bulletin 44N7 (2004) 25 [hep-th/0405016].

[6] C. Q. Geng, M. W. Hossain, R. Myrzakulov, M. Sami and E. N. Saridakis, Phys. Rev. D 92, no. 2, 023522 (2015) [arXiv:1502.03597 [gr-qc]].

[7] M. Wali Hossain, R. Myrzakulov, M. Sami and E. N. Saridakis, Int. J. Mod. Phys. D 24, no. 05, 1530014 (2015) [arXiv:1410.6100 [gr-qc]].

[8] Y. F. Cai, E. N. Saridakis, M. R. Setare and J. Q. Xia, Phys. Rept. 493, 1 (2010) [arXiv:0909.2776 [hep-th]].

[9] S. Capozziello ,M. De Laurentis,O. Luongo, A. C. Ruggeri, Galaxies, 1, 216, (2013).

[10] J. Martin, Comptes Rendus Physique 13566 (2012).

[11] S. Capozziello ,M. De Laurentis,O. Luongo, Int. J. Mod. Phys. D 24 , 1541002, (2014).

[12] H. A. Buchdahl, Mon. Not. R. Astr. Soc., 150, 1, (1970).

[13] K. Bamba, S. Nojiri, S. D. Odintsov, J. Cosmol. Astropart. Phys. 10, 045, (2008).

[14] J. Magueijo and L. Smolin, Class. Quant. Grav. 211725 (2004);J. J. Peng and S. Q. Wu, Gen. Rel. Grav. 40 2619 (2008).

[15] J. Magueijo and L. Smolin, Phys. Rev. Lett. 88190403 (2002) ;J. Magueijo and L. Smolin, Phys. Rev. D 71026010 (2005) ;J. L. Cortes and J. Gamboa, Phys. Rev. D 71065015 (2005).

[16] P. Rudra, Mir Faizal, Ahmed Farag Ali, Nucl. Phys. B 909, 725 (2016);Amani Ashour, Mir Faizal, Ahmed Farag Ali, Fayal Hammad , Eur.Phys.J. C76 (2016) no.5, 264; Ahmed Farag Ali, Mir Faizal, Barun Majumder, Ravi Mistry,Int.J.Geom.Meth.Mod.Phys. 12 (2015), 1550085 ;Ahmed Farag Ali, Mir Faizal, Mohammed M. Khalil , Nucl.Phys. B894 (2015) 341-360; Ahmed Farag Ali, Mir Faizal, Mohammed M. Khalil ,Phys.Lett. B743 (2015) 295; Ahmed Farag Ali, Mir Faizal, Mohammed M. Khalil ; JHEP 1412 (2014) 159 Ahmed Farag Ali, Mir Faizal, Barun Majumder;Europhys.Lett. 109 (2015) 20001.

[17] K. Hayashi, Phys. Lett. B69, 441 (1977).

[18] K. Hayashi and T. Shirafuji, Phys. Rev. D 19 (1979) 3524; 24, 3312 (1981).

[19] R. Weitzenbock, Invarianten Theorie. Nordhoff, Groningen (1923).

[20] Y. F. Cai, S. Capozziello, M. De Laurentis and E. N. Saridakis, Rept. Prog. Phys. 79, no. 10, 106901 (2016) [arXiv:1511.07586 [gr-qc]].

[21] R. Ferraro and F. Fiorini, Phys. Rev. D 84, 083518 (2011).

[22] D. Momeni and R. Myrzakulov, Astrophys. Space Sci. 360 (2015) 28 [arXiv:1511.01205 [physics.gen-ph]].

[23] D. Momeni, R. Myrzakulov, L. Sebastiani and M. R. Setare, Int. J. Geom. Meth. Mod. Phys. 12, 1550015 (2015) [arXiv:1210.7965 [hep-th]].

[24] D. Momeni, A. Altaibayeva and R. Myrzakulov, Int. J. Geom. Meth. Mod. Phys. 11, 1450091 (2014) [arXiv:1407.5662 $[\mathrm{gr}-\mathrm{qc}]]$.

[25] S. Chattopadhyay, A. Jawad, D. Momeni and R. Myrzakulov, Astrophys. Space Sci. 353 (2014) no.1, 279 [arXiv:1406.2307 [gr-qc]].

[26] D. Momeni and R. Myrzakulov, Int. J. Geom. Meth. Mod. Phys. 11 (2014) no.08, 1450077 [arXiv:1405.5863 [gr-qc]].

[27] D. Momeni and R. Myrzakulov, Eur. Phys. J. Plus 129 (2014) 137 [arXiv:1404.0778 [gr-qc]].

[28] M. Jamil, D. Momeni and R. Myrzakulov, Int. J. Theor. Phys. 54 (2015) no.4, 1098 [arXiv:1309.3269 [gr-qc]].

[29] M. E. Rodrigues, M. J. S. Houndjo, J. Tossa, D. Momeni and R. Myrzakulov, JCAP 1311 (2013) 024 [arXiv:1306.2280 $[\mathrm{gr}-\mathrm{qc}]]$.

[30] M. U. Farooq, M. Jamil, D. Momeni and R. Myrzakulov, Can. J. Phys. 91 (2013) 703 [arXiv:1306.1637 [astro-ph.CO]].

[31] M. E. Rodrigues, M. J. S. Houndjo, D. Momeni and R. Myrzakulov, Int. J. Mod. Phys. D 22 (2013) 1350043 [arXiv:1302.4372 [physics.gen-ph]].

[32] M. Jamil, D. Momeni and R. Myrzakulov, Eur. Phys. J. C 73 (2013) 2267 [arXiv:1212.6017 [gr-qc]].

[33] M. Jamil, D. Momeni and R. Myrzakulov, Gen. Rel. Grav. 45 (2013) 263 [arXiv:1211.3740 [physics.gen-ph]].

[34] M. Jamil, D. Momeni and R. Myrzakulov, Eur. Phys. J. C 72 (2012) 2137 [arXiv:1210.0001 [physics.gen-ph]].

[35] M. Jamil, D. Momeni and R. Myrzakulov, Eur. Phys. J. C 72 (2012) 2122 [arXiv:1209.1298 [gr-qc]]. 
[36] M. Jamil, D. Momeni and R. Myrzakulov, Eur. Phys. J. C 72 (2012) 2075 [arXiv:1208.0025 [gr-qc]].

[37] W. El Hanafy and G. G. L. Nashed, Astrophys. Space Sci. 361 (2016) no.8, 266 [arXiv:1510.02337 [gr-qc]].

[38] M. Jamil, K. Yesmakhanova, D. Momeni and R. Myrzakulov, Central Eur. J. Phys. 10 (2012) 1065 [arXiv:1207.2735 $[\mathrm{gr}-\mathrm{qc}]]$.

[39] M. J. S. Houndjo, D. Momeni and R. Myrzakulov, Int. J. Mod. Phys. D 21 (2012) 1250093 [arXiv:1206.3938 [physics.gen$\mathrm{ph}]$.

[40] M. I. Wanas, G. G. L. Nashed and O. A. Ibrahim, Chin. Phys. B 25 (2016) no.5, 050401.

[41] K. Bamba, G. G. L. Nashed, W. El Hanafy and S. K. Ibraheem, Phys. Rev. D 94 (2016) no.8, 083513 doi:10.1103/PhysRevD.94.083513 [arXiv:1604.07604 [gr-qc]].

[42] M. E. Rodrigues, M. J. S. Houndjo, D. Momeni and R. Myrzakulov, Int. J. Mod. Phys. D 22 (2013) 1350043 [arXiv:1302.4372 [physics.gen-ph]].

[43] M. Jamil, D. Momeni and R. Myrzakulov, Eur. Phys. J. C 72 (2012) 1959 [arXiv:1202.4926 [physics.gen-ph]].

[44] W. El Hanafy and G. G. L. Nashed, Astrophys. Space Sci. 361 (2016) no.2, 68 [arXiv:1507.07377 [gr-qc]].

[45] T., Phys. Rev. D 84, 024042 (2011).

[46] C. G. , A. Mussa and N. Tamanini, Class. Quant.Grav. 28, 245020 (2011).

[47] M. Hamani Daouda, M. E. Rodrigues and

[48] M. J. S. Houndjo, Eur. Phys. J. C 71, 1817 (2011).

[49] M. Camenzind, "Compact Objects in Astrophysics", Springer-Verlag Berlin Heidelberg (2007).

[50] R. C. Tolman. Proc. Nat. Acad. Sc., 20, 169, 1934.

[51] R. C. Tolman. Phys. Rev., 55, 364, 1939.

[52] J. R. Oppenheimer and G. M. Volkoff. Phys. Rev., 55, 374, 1939.

[53] A. V. Astashenok, S. Capozziello and S. D. Odintsov, arXiv:1401.4546 [gr-qc].

[54] G. Abbas, D. Momeni, M. A. Ali, R. Myrzakulov and S. Qaisar, arXiv:1501.00427 [gr-qc].

[55] A. V. Astashenok, S. Capozziello and S. D. Odintsov, JCAP 1312, 040 (2013) [arXiv:1309.1978 [gr-qc]].

[56] S. Nojiri., S. D. Odintsov, Phys.Lett. B631,1(2005).

[57] E. Chabanat, P. Bonche, P. Haensel, J. Meyer, R. Schaeffer, Nucl. Phys. A 635, 231 (1998).

[58] F. Douchin, P. Haensel, Phys. Lett. B 485, 107 (2000).

[59] F. Douchin, P. Haensel, Astron. and Astroph. 380, 151 (2001).

[60] V.R. Pandharipande, D.G. Ravenhall D.G., Hot nuclear matter in Nuclear Matter and Heavy Ion Collisions, Eds. M. Soyeur, H. Flocard, B.Tamain, and M. Porneuf (Dordrecht: Reidel), 103-132 (1989).

[61] S. Arapoglu, C. Deliduman, K. Yavuz Eksi, JCAP 1107, 020 (2011) [arXiv:1003.3179v3[gr-qc]].

[62] H. Alavirad, J.M. Weller, [arXiv:1307.7977v1[gr-qc]].

[63] S. Deser and R. P. Woodard, Phys. Rev. Lett. 99, 111301 (2007) [arXiv:0706.2151 [astro-ph]].

[64] C. Deffayet and R. P. Woodard, JCAP 0908, 023 (2009) [arXiv:0904.0961 [gr-qc]].

[65] S. Nojiri and S. D. Odintsov, Phys. Lett. B 659, 821 (2008) [arXiv:0708.0924 [hep-th]].

[66] S. Jhingan, S. Nojiri, S. D. Odintsov, M. Sami, I. Thongkool and S. Zerbini, Phys. Lett. B 663, 424 (2008) [arXiv:0803.2613 [hep-th]].

[67] K. Bamba, S. Nojiri, S. D. Odintsov and M. Sasaki, Gen. Rel. Grav. 44, 1321 (2012) [arXiv:1104.2692 [hep-th]].

[68] A. V. Astashenok, S. Capozziello and S. D. Odintsov, arXiv:1405.6663 [gr-qc].

[69] M. E. Rodrigues, M. J. S. Houndjo, J. Tossa, D. Momeni and R. Myrzakulov, JCAP 1311 (2013) 024 [arXiv:1306.2280 $[\mathrm{gr}-\mathrm{qc}]]$.

[70] C. G. Boehmer, T. Harko and F. S. N. Lobo, Phys. Rev. D 85, 044033 (2012) [arXiv:1110.5756 [gr-qc]].

[71] M. Jamil, D. Momeni and R. Myrzakulov, Eur. Phys. J. C 73 (2013) 2267 [arXiv:1212.6017 [gr-qc]].

[72] G. Abbas, G. Abbas, S. Qaisar, A. Jawad, S. Qaisar and A. Jawad, Astrophys. Space Sci. 359 (2015) no.2, 57 [arXiv:1509.06711 [physics.gen-ph]].

[73] G. Abbas, S. Qaisar and M. A. Meraj, Astrophys. Space Sci. 357 (2015) no.2, 156.

[74] M. Zubair and G. Abbas, Astrophys. Space Sci. 361 (2016) no.1, 27 [arXiv:1507.00247 [physics.gen-ph]].

[75] M. Zubair, Int. Journal of Modern Physics D25, 1650057(2016)

[76] M. Zubair et al. Astrophys Space Sci (2016) 361:8; M. Zubair and G. Abbas, Astrophys Space Sci (2016) 361:342

[77] G. Abbas, A. Kanwal and M. Zubair, Astrophys. Space Sci. 357 (2015) no.2, 109 [arXiv:1501.05829 [physics.gen-ph]].

[78] S. C. Ulhoa and P. M. M. Rocha, Braz. J. Phys. 43, 162 (2013) [arXiv:1206.3675 [gr-qc]].

[79] C. G. Boehmer, A. Mussa and N. Tamanini, Class. Quant. Grav. 28, 245020 (2011) [arXiv:1107.4455 [gr-qc]].

[80] R. Weitzenboc̈k, Invariantentheorie, Noordhoff, Groningen (1923).

[81] C. Deliduman and B. Yapiskan, arXiv:1103.2225 [gr-qc].

[82] J. M. Lattimer, A. W. Steiner , Astrophys. J. 784, 123 (2014) [arXiv:1305.3242 [astro-ph.HE]].

[83] X. D. Li , I. Bombaci ,M. Dey, J. Dey and E. P. J. van den Heuvel, Phys. Rev. Lett. 83, 3776 (1999) [hep-ph/9905356].

[84] D. A. Leahy, S. M. Morsink and C. Cadeau, Astrophys. J. 672, 1119 (2008) [astro-ph/0703287 [ASTRO-PH]].

[85] L. Herrera, Phys. Lett. A165, 206(1992) 